\title{
Obtaining Robust Models from Imbalanced Data
}

\author{
Wentao Wang \\ Michigan State University \\ East Lansing, Michigan, USA \\ wangw116@msu.edu
}

\begin{abstract}
The vulnerability of deep neural network (DNN) models has been verified by the existence of adversarial examples. By exploiting slight changes to input examples, the generated adversarial examples can easily cause well trained DNN models make wrong predictions. Many defense methods have been proposed to improve the robustness of DNN models against adversarial examples. Among them, adversarial training has been empirically proven to be one of the most effective methods. Almost all existing studies about adversarial training are focused on balanced datasets, where each class has an equal amount of training examples. However, as datasets collected in real-world applications cannot guarantee all contained classes are uniformly distributed, it would be much challenging to obtain robust models in those real applications where the available training datasets are imbalanced. As the initial effort to study this problem, we first investigate the different behaviors between adversarially trained models and naturally trained models using imbalanced training datasets and then explore possible solutions to facilitate adversarial training under imbalanced settings.
\end{abstract}

\section{KEYWORDS}

imbalanced data, deep neural networks, model robustness

\section{ACM Reference Format:}

Wentao Wang. 2022. Obtaining Robust Models from Imbalanced Data. In Proceedings of the Fifteenth ACM International Conference on Web Search and Data Mining (WSDM '22), February 21-25, 2022, Tempe, AZ, USA. ACM, New York, NY, USA, 2 pages. https://doi.org/10.1145/3488560.3502217

\section{MOTIVATION}

The deep neural network (DNN) models have achieved great success in many application scenarios, ranging from image classification $[14,19]$ to machine translation $[16,20]$. However, the existence of adversarial examples $[9,21]$ has risen huge concerns on applying DNN models into security-critical applications, such as autonomous driving [6]. As one countermeasure against adversarial examples, adversarial training $[17,24]$ has been empirically proven to be one of the most effective defense methods. Briefly, it can be formulated as solving a min-max optimization problem, where a DNN model is trained on minimizing the prediction error on adversarial examples generated by iteratively maximizing some loss function.

Permission to make digital or hard copies of part or all of this work for personal or classroom use is granted without fee provided that copies are not made or distributed for profit or commercial advantage and that copies bear this notice and the full citation on the first page. Copyrights for third-party components of this work must be honored.

For all other uses, contact the owner/author(s).

WSDM '22, February 21-25, 2022, Tempe, AZ, USA

(C) 2022 Copyright held by the owner/author(s).

ACM ISBN 978-1-4503-9132-0/22/02.

https://doi.org/10.1145/3488560.3502217
Although promising to improve the model's robustness, almost all existing adversarial training methods $[22,24]$ assume that the number of training examples from each class is equally distributed. However, datasets collected from real-world applications typically have imbalanced distribution $[8,15]$. Previous works have demonstrated that common machine learning methods could perform worse when training on the imbalanced datasets $[2,12]$. Hence, it is natural to ask: what is the behavior of adversarial training under imbalanced scenarios? In addition, if the performance of adversarial training methods are also dropped, a following question is: Can we directly apply existing imbalanced learning strategies in natural training to tackle the imbalance issue for adversarial training?

\section{RELATE WORK}

Our study is closely related to imbalanced learning and adversarial robustness.

Imbalanced Learning. Most existing works of imbalanced learning can be roughly classified into two categories, i.e., re-sampling and reweighting. Re-sampling methods aim to reduce the level of imbalance through either over-sampling data examples from underrepresented classes $[3,4]$ or under-sampling data examples from well-represented classes $[7,10]$. Reweighting methods allocate different weights for different classes or even different data examples. More information about imbalanced learning can be found in recent surveys $[11,12]$. Note that, the majority of existing methods focused on the nature training scenario and their trained models will be crashed when facing adversarial attacks $[9,21]$.

Adversarial Robustness. To improve model robustness against adversarial attacks, various defense methods have been proposed [ 9 , 17, 18]. Among them, adversarial training has been proven to be one of the most effective methods [1]. Adversarial training can be formulated as solving a min-max optimization problem where the outer minimization process enforces the model to be robust to adversarial examples, generated by the inner maximization process via some existing attacking methods like PGD [17]. More details about adversarial robustness can be found in recent surveys [ 5 , 23]. Since almost all studies of adversarial training are focused on balanced datasets, it's worthwhile to investigate the performance of adversarial training methods on imbalanced training datasets.

\section{METHODOLOGY}

\subsection{Preliminary Study}

As the initial effort to study the imbalanced problem in adversarial training, we start our research from exploring answers for aforementioned two questions. Through our preliminary experiments conducted on an imbalanced training dataset constructed from CIFAR10 [13] dataset, we observe that, 1) compared to naturally trained models, adversarially trained models always present very 
low standard accuracy and robust accuracy on under-represented classes; and 2) in adversarial training, increasing weights for an under-represented class can substantially improve the standard accuracy and robust accuracy on this class, but drastically hurt the model's performance on the well-represented class. These observations indicate that neither adversarial training methods nor adversarial training methods equipping with the reweighting strategy can handle the imbalance issue and new solutions are expected to solve this problem.

\subsection{Proposed Solution}

By integrating the reweighting strategy into adversarial training, the reweighted adversarial training can be formulated as

$$
\min _{\theta} \frac{1}{n} \sum_{i=1}^{n} \max _{\left\|\mathbf{x}_{i}^{\prime}-\mathbf{x}_{i}\right\|_{p} \leq \epsilon} w_{i} \mathcal{L}\left(f_{\theta}\left(\mathbf{x}_{i}^{\prime}\right), y_{i}\right),
$$

where $w_{i}$ is a weight value assigned for each input example $\left(\mathbf{x}_{i}, y_{i}\right)$ based on the example size of the class $\left(\mathbf{x}_{i}, y_{i}\right)$ belongs to or some properties of $\left(\mathbf{x}_{i}, y_{i}\right)$, and $\mathcal{L}(\cdot, \cdot)$ denotes a loss function.

To facilitate the reweighting strategy in adversarial training under imbalanced scenarios, we propose a novel feature separation loss. We aim to enforce the learned feature space as separable as possible. More specifically, our goal is to make 1) the learned features of examples from the same class well clustered, and 2) the features of examples from different classes well separated. By achieving this goal, the model is able to learn more discriminative features for each class. The feature separation loss is formally defined as:

$$
\mathcal{L}_{\text {sep }}\left(\mathbf{x}_{i}^{\prime}\right)=-\frac{1}{|P(i)|} \sum_{p \in P(i)} \log \frac{\exp \left(\mathbf{z}_{i}^{\prime} \cdot \mathbf{z}_{p}^{\prime} / \tau\right)}{\sum_{a \in A(i)} \exp \left(\mathbf{z}_{i}^{\prime} \cdot \mathbf{z}_{a}^{\prime} / \tau\right)},
$$

where $\mathbf{z}_{i}^{\prime}$ is the feature representation of the adversarial example $\mathbf{x}_{i}^{\prime}$ of $\mathbf{x}_{i}, \tau \in \mathcal{R}^{+}$is a scalar temperature parameter, $P(i)$ denotes the set of input examples belonging to the same class with $\mathbf{x}_{i}$ and $A(i)$ indicates the set of all input examples excepts $\mathbf{x}_{i}^{\prime}$.

By combining the feature separation loss with the reweighted adversarial training, the final object function for our proposed method can be defined as:

$$
\min _{\theta} \frac{1}{n} \sum_{i=1}^{n} \max _{\left\|\mathbf{x}_{i}^{\prime}-\mathbf{x}_{i}\right\|_{p} \leq \epsilon} w_{i} \mathcal{L}\left(f_{\theta}\left(\mathbf{x}_{i}^{\prime}\right), y_{i}\right)+\lambda \mathcal{L}_{\text {sep }}\left(\mathbf{x}_{i}^{\prime}\right),
$$

where the hyper-parameter $\lambda$ is used to balance the contributions from two different terms.

\section{FUTURE DIRECTIONS}

Our current reweighting based solution can effectively handle the imbalanced issue and obtain robust models from imbalanced training datasets. In the future, we plan to investigate whether other existing imbalanced learning methods proposed in natural training scenarios can help training robust models under imbalanced settings. For example, we are interested in exploring whether some existing re-sampling strategies can be utilized as a data augmentation tool to solve this problem. As shown in our preliminary study, adversarially trained models reveal different behaviors with naturally trained models, we guess existing re-sampling strategies also cannot be integrated into adversarial training directly and, hence, extra efforts are needed.

\section{ACKNOWLEDGMENTS}

The author would like to thank his Ph.D. advisor Dr. Jiliang Tang for constructive suggestions. Wentao Wang and Dr. Jiliang Tang are supported by the National Science Foundation (NSF) under grant numbers IIS1714741, CNS1815636, IIS1845081, IIS1907704, DRL2025244, IIS1928278, IIS1955285, IOS2107215, IOS2035472 and Army Research Office (ARO) under grant number W911NF-21-10198.

\section{REFERENCES}

[1] Anish Athalye, Nicholas Carlini, and David Wagner. 2018. Obfuscated gradients give a false sense of security: Circumventing defenses to adversarial examples. In International Conference on Machine Learning. PMLR, 274-283.

[2] Paula Branco, Luis Torgo, and Rita Ribeiro. 2015. A survey of predictive modelling under imbalanced distributions. arXiv preprint arXiv:1505.01658 (2015).

[3] Mateusz Buda, Atsuto Maki, and Maciej A Mazurowski. 2018. A systematic study of the class imbalance problem in convolutional neural networks. Neural Networks 106 (2018), 249-259.

[4] Jonathon Byrd and Zachary Lipton. 2019. What is the effect of importance weighting in deep learning?. In International Conference on Machine Learning. PMLR, 872-881.

[5] Anirban Chakraborty, Manaar Alam, Vishal Dey, Anupam Chattopadhyay, and Debdeep Mukhopadhyay. 2018. Adversarial attacks and defences: A survey. arXiv preprint arXiv:1810.00069 (2018).

[6] Chenyi Chen, Ari Seff, Alain Kornhauser, and Jianxiong Xiao. 2015. Deepdriving: Learning affordance for direct perception in autonomous driving. In Proceedings of the IEEE international conference on computer vision. 2722-2730.

[7] Chris Drummond, Robert C Holte, et al. 2003. C4. 5, class imbalance, and cost sensitivity: why under-sampling beats over-sampling. In Workshop on learning from imbalanced datasets II, Vol. 11. Citeseer, 1-8.

[8] Mark Everingham, Luc Van Gool, Christopher KI Williams, John Winn, and Andrew Zisserman. 2010. The pascal visual object classes (voc) challenge. International journal of computer vision 88, 2 (2010), 303-338.

[9] Ian J Goodfellow, Jonathon Shlens, and Christian Szegedy. 2014. Explaining and harnessing adversarial examples. arXiv preprint arXiv:1412.6572 (2014).

[10] Haibo He and Edwardo A Garcia. 2009. Learning from imbalanced data. IEEE Transactions on knowledge and data engineering 21, 9 (2009), 1263-1284.

[11] Haibo He and Yunqian Ma. 2013. Imbalanced learning: foundations, algorithms, and applications. (2013).

[12] Justin M Johnson and Taghi M Khoshgoftaar. 2019. Survey on deep learning with class imbalance. Journal of Big Data 6, 1 (2019), 1-54.

[13] Alex Krizhevsky, Geoffrey Hinton, et al. 2009. Learning multiple layers of features from tiny images. (2009).

[14] Alex Krizhevsky, Ilya Sutskever, and Geoffrey E Hinton. 2012. Imagenet classification with deep convolutional neural networks. Advances in neural information processing systems 25 (2012), 1097-1105.

[15] Tsung-Yi Lin, Michael Maire, Serge Belongie, James Hays, Pietro Perona, Deva Ramanan, Piotr Dollár, and C Lawrence Zitnick. 2014. Microsoft coco: Common objects in context. In European conference on computer vision. Springer, 740-755.

[16] Minh-Thang Luong, Hieu Pham, and Christopher D Manning. 2015. Effective approaches to attention-based neural machine translation. arXiv preprint arXiv:1508.04025 (2015).

[17] Aleksander Madry, Aleksandar Makelov, Ludwig Schmidt, Dimitris Tsipras, and Adrian Vladu. 2017. Towards deep learning models resistant to adversarial attacks. arXiv preprint arXiv:1706.06083 (2017).

[18] Aditi Raghunathan, Jacob Steinhardt, and Percy Liang. 2018. Certified defenses against adversarial examples. arXiv preprint arXiv:1801.09344 (2018).

[19] Karen Simonyan and Andrew Zisserman. 2014. Very deep convolutional networks for large-scale image recognition. arXiv preprint arXiv:1409.1556 (2014).

[20] Ilya Sutskever, Oriol Vinyals, and Quoc V Le. 2014. Sequence to sequence learning with neural networks. In Advances in neural information processing systems. 31043112.

[21] Christian Szegedy, Wojciech Zaremba, Ilya Sutskever, Joan Bruna, Dumitru Erhan, Ian Goodfellow, and Rob Fergus. 2013. Intriguing properties of neural networks. arXiv preprint arXiv:1312.6199 (2013).

[22] Yisen Wang, Difan Zou, Jinfeng Yi, James Bailey, Xingjun Ma, and Quanquan Gu. 2019. Improving adversarial robustness requires revisiting misclassified examples. In International Conference on Learning Representations.

[23] Han Xu, Yao Ma, Hao-Chen Liu, Debayan Deb, Hui Liu, Ji-Liang Tang, and Anil K Jain. 2020. Adversarial attacks and defenses in images, graphs and text: A review. International fournal of Automation and Computing 17, 2 (2020), 151-178.

[24] Hongyang Zhang, Yaodong Yu, Jiantao Jiao, Eric Xing, Laurent El Ghaoui, and Michael Jordan. 2019. Theoretically principled trade-off between robustness and accuracy. In International Conference on Machine Learning. PMLR, 7472-7482. 